\title{
Prevalence of CCHF Virus in Ticks and People and Public Awareness in Zhambyl Region, Kazakhstan
}

\author{
Jennifer R. Head ${ }^{\star 2,4}$, Yekatarina Bumburidi ${ }^{1}$, Stephanie J. Salyer ${ }^{2}$, Barbara Knust ${ }^{2}$, \\ Mirzabekova G. Kuralbekovna ${ }^{3}$ and Daphne B. Moffett ${ }^{1}$
}

${ }^{1} \mathrm{CDC}$-Central Asia Office, Almaty, Kazakhstan; ${ }^{2}$ Centers for Disease Control and Prevention, Atlanta, GA, USA; ${ }^{3} \mathrm{Zhambyl}$ Oblast

Department of Public Health, Taraz, Kazakhstan; ${ }^{4}$ Public Health Institute, San Francisco, CA, USA

\section{Objective \\ As part of CDC's Global Disease Detection work, in conjunction with Zhambyl Region Department of Health, we conducted a tick survey and human seroprevalence Knowledge, Attitudes, and Practices (KAP) survey of livestock-owning households in Zhambyl to assess $\mathrm{CCHF}$ seroprevalence and risk factors.}

\section{Introduction}

Crimean Congo Hemorrhagic Fever (CCHF) virus is a tick-borne pathogen that causes severe disease in people, with a distribution that extends from central Asia to southern Africa. In addition to tick bites, contact with bodily fluids from viremic livestock or from symptomatic humans are risk factors for infection. From 2000 to 2013, 73 cases of CCHF were reported in Zhambyl Region, Kazakhstan. CCHF virus is categorized as an "especially dangerous pathogen" in Kazakhstan and $\mathrm{CCHF}$ is prioritized for surveillance and treatment. Little is known about the seroprevalence of infection by CCHF virus in Zhambyl in ticks or people, and knowledge of risk factors for transmission of CCHF virus among at-risk populations is believed to be low.

\section{Methods}

Rural villages were classified as "endemic" or "non-endemic", where endemic areas reported $\geq 1$ CCHF case or a CCHF viruspositive tick in the past 5 years. In each group, 15 villages were chosen by population proportional to livestock population size. Livestock-owning households $(\mathrm{n}=969)$ were selected randomly from veterinary registries. One adult was randomly selected per house and ticks were collected from one randomly selected sheep or cow over 1 year of age per house. Data were weighted accounting for sampling design and analyzed in R.

\section{Results}

KAP surveys were completed for 950 people (98\%); of those, 923 (97\%) submitted blood for ELISA testing using Vector-Best Kits. Median age of human respondents was 46 years (range: $19-90$ ); $54 \%$ were male. Three individuals were anti-CCHF IgM positive, 12 anti-CCHF IgG positive and two positive for both. Weighted seroprevalence of CCHF in Zhambyl was $1.6 \%$ (95\% CI: 0.9, 3.0). In endemic villages, seroprevalence was $1.8 \%(95 \%$ CI: $1.0,3.0)$, compared to $1.2 \%$ (95\% CI: $0.4,4.0)$ in non-endemic villages. Of the 17 seropositive for CCHF, median age was 54 years; $58 \%$ were male. None reported previous CCHF diagnosis or illness with fever and hemorrhaging in the past five years. None reported high-risk tick exposure in the past four months. Controlling for age and sex, milking animals, an activity in which $40.3 \%$ of the population had engaged, was associated with infection in Poisson regression (OR: 2.53, 95\% CI: 1.27, 4.81). Of respondents who had heard of CCHF $(\mathrm{n}=791), 99.8 \%$ knew transmission was caused by a tick bite; few identified contact with animal blood $(8.2 \%)$ or tick crushing $(20.8 \%)$ as potential causes. Of the five seropositive by IgM, four participated in at least one of the following activities in the last four months: milking $(n=3)$, birthing $(n=2)$, shearing and slaughtering $(n=1)$. One reported experiencing an illness with joint pain within the past four months. Three were from non-endemic villages.

Entomologists inspected 465 cows and 528 sheep for ticks. Ticks were found on $61.5 \%$ (95\% CI: 48.1, 73.2) of cows $(n=254)$ and $46.3 \%(95 \%$ CI: $24.3,69.8)$ of sheep $(n=264)$. Ticks were grouped into pools by animal source and species. Over ninety-seven percent of the tick pools were from the family Ixoidadae, with the remaining from family Argasidae. The genus Hyalomma accounted for $65.8 \%$ of tick pools, Rhipicephalus for $31.8 \%$, Ornithodoros for 2.4\%, and Argas for 0.5\%. Pools contained an average of 4.5 ticks (range: $1-26$ ). Ticks were stored live at $4^{\circ} \mathrm{C}$ for up to 24 hours before being crushed and extracts tested for CCHF virus by PCR and Antigen testing. Of the 155 pools tested, seven $(2.4 \%, 95 \%$ CI: 1.1 , 5.0) were positive for CCHF virus by either PCR $(n=5)$ and/or antigen testing $(\mathrm{n}=4)$. A CCHF virus-positive tick was found on $1.4 \%(95 \%$ CI: $0.4,4.8)$ of all sheep and $4.8 \%$ (95\% CI: $2.3,10.0)$ of all cows. All CCHF virus-positive ticks were hard ticks of family Ixodidae, belonging to either genus Hyalomma $(\mathrm{n}=5)$ or Rhipicephalus $(\mathrm{n}=2)$. Two pools were from non-endemic villages.

\section{Conclusions}

Presence of CCHF virus-positive ticks and CCHF-seropositive humans in non-endemic areas may suggest a wider range of virus circulation. These findings will be used to inform and target public health messaging.

\section{Keywords}

CCHF; serosurvey; Kazakhstan; ticks; livestock

\author{
*Jennifer R. Head \\ E-mail: jrhead6@gmail.com
}

